\title{
Muscle Contributions to Pre-Swing Biomechanical Tasks Influence Swing Leg Mechanics in Individuals Post-Stroke during Walking
}

\section{Lydia G. Brough}

The University of Texas at Austin

Steven A. Kautz

Medical University of South Carolina

Richard Neptune ( $\sim$ rneptune@mail.utexas.edu )

The University of Texas at Austin https://orcid.org/0000-0003-2425-004X

\section{Research}

Keywords: Stiff knee gait, modeling, gait, compensation, biomechanics

Posted Date: November 9th, 2021

DOI: https://doi.org/10.21203/rs.3.rs-1040994/v1

License: (c) (1) This work is licensed under a Creative Commons Attribution 4.0 International License.

Read Full License 


\section{Abstract \\ Background}

Successful walking requires the execution of the pre-swing biomechanical tasks of body propulsion and leg swing initiation, which are often impaired post-stroke. While excess rectus femoris activity during swing is often associated with low knee flexion, previous work has suggested that deficits in propulsion and leg swing initiation may also contribute. The purpose of this study was to determine underlying causes of propulsion, leg swing initiation and knee flexion deficits in pre-swing and their link to stiff knee gait in stroke survivors.

\section{Methods}

Musculoskeletal models and forward dynamic simulations were developed for individuals post-stroke $(n=15)$ and neurotypical participants $(n=5)$. Linear regressions were used to evaluate the relationships between peak knee flexion, braking and propulsion symmetry, and individual muscle contributions to braking, propulsion, knee flexion in pre-swing, and leg swing initiation.

\section{Results}

$27 \%$ of individuals post-stroke had higher plantarflexor contributions to propulsion and $47 \%$ had higher vasti contributions to braking on their paretic leg relative to their nonparetic leg. Higher gastrocnemius contributions to propulsion were correlated to paretic propulsion symmetry $(p=0.005)$ while soleus contributions were not. Higher vasti contributions to braking in pre-swing predicted lower knee flexion $(p=0.022)$. The rectus femoris and iliopsoas did not directly contribute to lower knee flexion acceleration in pre-swing compared to contributions from the vasti. However, for some individuals with low knee flexion, during pre-swing the rectus femoris absorbed more power and the iliopsoas contributed less power to the paretic leg. Total muscle-tendon work done on the paretic leg in pre-swing was not correlated to knee flexion during swing.

\section{Conclusions}

These results emphasize the multiple causes of propulsion asymmetry in individuals post-stroke, including low plantarflexor contributions to propulsion, increased vasti contributions to braking and reliance on compensatory mechanisms. The results also show that the rectus femoris is not a major contributor to knee flexion in pre-swing, but absorbs more power from the paretic leg in pre-swing in some individuals with stiff knee gait. These results further highlight the heterogeneity of the post-stroke population and the need to identify individual causes of propulsion and knee flexion deficits to improve rehabilitation outcomes. 


\section{Background}

Over 795,000 people in the United States experience a stroke each year and over half of stroke survivors over age 65 have reduced mobility [1]. Regaining walking ability is an important goal of rehabilitation as walking speed is a critical predictor of long-term health [2] and stroke survivors who achieve limited or full community walking speeds report an overall higher quality of life than those who remain household ambulators [3]. Successful walking requires the execution of the critical pre-swing biomechanical subtasks of body propulsion and leg swing initiation, which are often impaired post-stroke [4,5].

Deficits in these late stance subtasks may also have important implications for achieving adequate knee flexion during the swing phase. For example, a lack of push-off acceleration rather than over-activity of the knee extensors may be the primary cause of stiff knee gait in some individuals post-stroke [2] and stimulating the plantarflexors in pre-swing increased peak knee flexion [6]. In addition, late stance phase braking forces in stroke survivors predict less knee flexion during swing, and late braking and low propulsion have been shown to predict circumduction [7]. However, interestingly the total propulsive force is not correlated to knee flexion [7] and it is unknown if the relationships between braking, propulsion and swing phase kinematics are causal or correlative.

Braking and propulsion deficits are common in individuals post-stroke [4]. While healthy individuals produce braking only in the first half of stance and propulsion in the second half, stroke survivors often have prolonged braking and low propulsion output, which predicts slower walking speed $[8,9]$. The plantarflexor muscles are primary contributors to propulsion $[10,11]$ and decreased plantarflexor contributions to propulsion have been observed in stroke survivors [12-14], with post-training increases in walking speed being predicted by greater plantarflexor contributions to propulsion [15]. However, a lack of propulsion can occur not only because of low plantarflexor output, but propulsion can be offset by prolonged activity from the vasti, which are primary contributors to braking forces [16]. On average, individuals with impaired plantarflexor coordination do not have lower propulsion, but rather greater braking than individuals with normal plantarflexor coordination [17], which is likely due to co-activation of the plantarflexors and vasti muscles. Due to the characteristically high variability between stroke survivors, there are a number of mechanisms that can cause the propulsion deficits.

Stroke survivors also experience deficits in leg movement throughout swing [18, 19], with modeling studies having identified knee flexion velocity at toe-off as the primary contributor to peak knee flexion during swing [20-22]. Knee velocity at toe-off may be diminished by late braking forces because muscles such as the vasti and rectus femoris that contribute to braking also contribute to knee extension and oppose leg swing initiation in late-stance [16]. Deficits in leg swing initiation may also be caused by decreased gastrocnemius contributions to leg-swing initiation, leading to lower knee velocity at toe-off and consequently less knee flexion during swing. For example, medial gastrocnemius contribution to knee flexion acceleration increased after gait retraining [15]. However, a representative stroke survivor with a limited community walking speed had similar contributions from the gastrocnemius and vasti compared to a healthy control walking at the same speed but less power delivered to the leg in pre-swing 
by the iliopsoas [14]. Thus, the high degree of variability in stroke survivors makes these results difficult to generalize and it is unknown how these deficits in leg-swing initiation impact swing phase kinematics.

Impaired knee flexion is often attributed to rectus femoris activity [18, 23]. In a previous modeling study, eliminating rectus femoris activity in pre-swing was more effective than eliminating rectus femoris activity in early swing for improving knee flexion [24]. The gluteus medius, vasti, and rectus femoris have the greatest potential to decrease knee flexion velocity in late stance, while the sartorius, gracilis, biceps femoris short head, gastrocnemius, iliopsoas and hamstrings have the greatest potential to increase knee flexion velocity in late stance [25], although it is unknown which muscles most affect pre-swing knee flexion velocity in stroke survivors.

Previous work has established the importance of pre-swing conditions to achieving adequate swing phase knee flexion. However, actual muscle contributions to propulsion, knee velocity and leg-swing initiation in stroke survivors and their relationship to swing-phase knee flexion has not been established. Moreover, most simulation studies are limited by a low number of participants, and therefore may not be generalizable to the overall stroke population. Thus, the objectives of this study were to: 1) analyze a large number of stroke survivors and determine the underlying causes of propulsion and braking deficits in late-stance, 2) identify primary muscle contributors to knee velocity and leg power in late stance, and 3 ) determine whether these muscle contributions to knee velocity and leg power predict knee flexion in swing. We hypothesized that 1) braking and propulsion asymmetries would be caused by both low plantarflexor contributions to propulsion and high vasti contributions to braking, 2) vasti and plantarflexor contributions to propulsion and braking in pre-swing would predict swing phase knee flexion, 3 ) the rectus femoris would be a major contributor to knee extension in pre-swing in individuals with stiff knee gait, and 4) greater knee flexion would be correlated with greater power delivered to the leg in pre-swing. The outcomes of this work will highlight specific deficits in propulsion and leg swing initiation post-stroke and their implications for swing phase knee flexion, which will provide a basis for developing targeted walking interventions.

\section{Methods}

\section{Data collection}

Kinematic, kinetic and electromyography data were collected from 15 stroke survivors ( 6 female, age: $56.1 \pm 13.3$ years, at least six months post-stroke) and 5 age-similar control subjects ( 2 female, age: 53.4 \pm 7.3 years) (Table 1). Participants provided informed written consent to the Institutional Review Board approved protocol. Participants walked on a split-belt instrumented treadmill (Bertec, Columbus, Ohio) at their self-selected walking speed. Before data collection was initiated, participants practiced treadmill walking to get comfortable with the experimental setup and walked for at least 10 seconds to reach steady-state before each 30 -second trial. Kinematic data were collected at $120 \mathrm{~Hz}$ using a 12-camera motion capture system and 65 active markers (PhaseSpace, San Leandro, CA). Electromyography (EMG) data were collected (Motion Labs, Cortlandt, NY) at $1000 \mathrm{~Hz}$ from bilateral electrodes placed on the 
medial gastrocnemius, soleus, vastus medialis, lateral hamstrings, medial hamstrings, rectus femoris and tibialis anterior. Kinematic and ground reaction force (GRF) data were low-pass filtered at $6 \mathrm{~Hz}$ and $15 \mathrm{~Hz}$, respectively. EMG data were high-pass filtered at $40 \mathrm{~Hz}$, demeaned, rectified and low-pass filtered at $4 \mathrm{~Hz}$.

\section{Musculoskeletal models \& simulations}

A representative paretic leg gait cycle (left gait cycle for control subjects) for each participant was chosen for further analysis using the functional medial distance depth method [26]. Using OpenSim 3.3 [27], a musculoskeletal model with 23 degrees of freedom and 92 muscle actuators [28] was scaled to match the anthropometry of each participant. An inverse kinematics analysis estimated generalized coordinates during the selected gait cycle by minimizing the difference between experimental and model markers [27]. To reduce dynamic inconsistencies between the experimental GRFs and body segment kinematics, a residual reduction algorithm (RRA) fine-tuned the torso center of mass (COM) position, segment masses and joint kinematics [27] until residual forces and tracking errors were within acceptable ranges [29]. Computed muscle control (CMC) [30] then estimated muscle excitations that reproduced the experimental motion while minimizing the sum of excitations squared. Muscle excitation timing was constrained to approximately follow normalized EMG signals [31].

\section{Data analysis}

Body propulsion and braking were calculated from the integral of the anterior and posterior GRF, respectively. Propulsion and braking asymmetries were defined as the percentage of paretic propulsion (PP) and paretic braking (PB), i.e., the paretic propulsive or braking impulse divided by the sum of paretic and nonparetic propulsive or braking impulses (e.g., perfectly symmetric $P P=0.5$ ). Heel strike and toe-off were identified from GRFs using a threshold of $20 \mathrm{~N}$. Knee flexion velocity at toe-off and peak knee flexion during swing were identified using joint kinematics from RRA. Pre-swing was defined as the double support phase between nonparetic (right) heel strike and paretic (left) toe-off. Individuals were placed into low or typical knee flexion groups depending on whether differences between the paretic and nonparetic

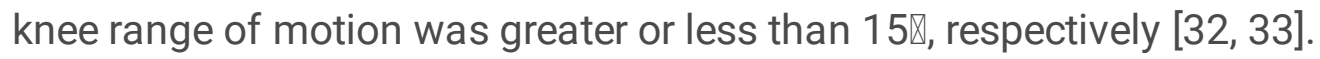

\section{Muscle contributions to biomechanical subtasks}

Induced acceleration and segmental power analyses [34] were used to determine individual muscle contributions to braking, propulsion, knee flexion in pre-swing and leg swing initiation. Muscle contributions were then analyzed in functional groups (Table 2). To perform the induced acceleration analyses, a surface rolling constraint was applied to the feet during stance [35] and muscle forces were determined using activations from CMC. Results were compared to experimental GRFs to ensure that the acceleration of the COM tracked the normalized GRFs. Muscle contributions to braking and propulsion were defined as each muscle's contribution to the anteroposterior (AP) acceleration of the body's COM integrated with respect to time over stance, normalized by walking speed. Muscle contributions to knee flexion during pre-swing were determined by integrating each muscle's contribution to knee flexion acceleration over time. To determine muscle contributions to leg swing initiation, a segment power analysis was used to determine the power delivered, absorbed or transferred to the leg by each muscle 
[16]. Muscle power was integrated over time to determine each muscle's mechanical work on the leg during pre-swing and was analyzed with and without normalizing by walking speed. Results were normalized by walking speed because walking speed is correlated with AP GRFs, knee flexion, and muscle power. However, results for muscle work were also presented without normalization so the reader can interpret absolute muscle work in addition to work relative to walking speed.

\section{Statistical Analyses}

To test the hypotheses that greater vasti contributions to braking and lower plantarflexor contributions to propulsion would predict braking and propulsion asymmetries, linear regression models were created with PP and PB as the dependent measures and either soleus, gastrocnemius or vasti contributions to AP $\mathrm{COM}$ acceleration over stance as the independent measure. To test the hypothesis that pre-swing braking and propulsion would predict swing-phase knee flexion, linear regression models were created with peak knee flexion as the dependent measure and either total pre-swing AP GRF impulse (normalized by subject mass and walking speed), soleus, gastrocnemius or vasti contributions to AP COM acceleration in preswing (normalized by walking speed) as the independent measures. Similarly, to test the hypothesis that individuals with low knee flexion would have less work performed on the paretic leg by paretic muscles, a linear regression was created with peak knee flexion as the dependent measure and net muscle-tendon work performed on the paretic leg as the independent measure. Significance was defined as $a=0.05$. 
Table 1

Participant characteristics

\begin{tabular}{|c|c|c|c|c|}
\hline \multicolumn{5}{|c|}{ Stroke Survivors } \\
\hline Participant & Age & Mass (kg) & Treadmill Self-Selected Walking Speed $(\mathrm{m} / \mathrm{s})$ & Sex \\
\hline 1 & 75 & 66.6 & 0.44 & M \\
\hline 2 & 67 & 76.1 & 0.55 & $\mathrm{~F}$ \\
\hline 3 & 58 & 76.8 & 0.55 & $\mathrm{~F}$ \\
\hline 4 & 51 & 85.9 & 0.55 & M \\
\hline 5 & 53 & 112.7 & 0.35 & $\mathrm{~F}$ \\
\hline 6 & 63 & 114.6 & 0.40 & M \\
\hline 7 & 49 & 93.5 & 0.40 & M \\
\hline 8 & 70 & 85.0 & 0.30 & M \\
\hline 9 & 70 & 86.4 & 0.30 & M \\
\hline 10 & 55 & 53.7 & 0.30 & $\mathrm{~F}$ \\
\hline 11 & 60 & 75.8 & 0.45 & M \\
\hline 12 & 35 & 63.7 & 0.50 & $\mathrm{~F}$ \\
\hline 13 & 66 & 98.5 & 0.40 & M \\
\hline 14 & 26 & 77.7 & 0.30 & $\mathrm{~F}$ \\
\hline 15 & 43 & 84.9 & 0.20 & M \\
\hline Average & $56.1 \pm 13.3$ & $83.5 \pm 16.2$ & $0.4 \pm 0.1$ & \\
\hline \multicolumn{5}{|c|}{ Control Participants } \\
\hline Participant & Age & Mass (kg) & Walking Speed (m/s) & Sex \\
\hline C1 & 59 & 81.3 & 0.55 & M \\
\hline C2 & 40 & 79.7 & 1.10 & M \\
\hline C3 & 51 & 83.6 & 0.70 & $\mathrm{~F}$ \\
\hline C4 & 59 & 80.1 & 0.50 & $M$ \\
\hline C5 & 58 & 65.3 & 0.80 & $\mathrm{~F}$ \\
\hline Average: & $53.4 \pm 7.3$ & $78.0 \pm 6.5$ & $0.7 \pm 0.2$ & \\
\hline
\end{tabular}


Table 2

Muscle analysis groups.

\begin{tabular}{|ll|}
\hline Muscle Group & Muscles \\
\hline IL & Iliacus, Psoas \\
\hline AL & Adductor Longus, Adductor Brevis, Pectineus, Quadratus Femoris \\
\hline SAR & Superior, Middle, Inferior Adductor Magnus \\
\hline RF & Sartorius \\
\hline VAS & Rectus Femoris \\
\hline GMEDA & Vastus Medialis, Vastus Intermedius, Vastus Lateralis \\
\hline GMEDP & Posterior Gluteus Medius, Posterior Gluteus Minimus \\
\hline TFL & Tensor Fasciae Latae \\
\hline GMAX & Superior, Middle and Inferior Gluteus Maximus \\
\hline HAM & Semimembranosus, Semitendinosus, Biceps Femoris Long Head, Gracilis \\
\hline BFSH & Biceps Femoris Short Head \\
\hline GAS & Medial Gastrocnemius, Lateral Gastrocnemius \\
\hline SOL & Soleus, Tibialis Posterior, Flexor Digitorum Longus \\
\hline TA & Tibialis Anterior, Extensor Digitorum Longus \\
\hline
\end{tabular}

\section{Results}

Muscle contributions to braking and propulsion asymmetry

There was no correlation between propulsion asymmetry and vasti or soleus contributions to propulsion (Figure 1A \& 1C). However, gastrocnemius contributions to propulsion were positively associated with propulsion asymmetry in stroke survivors (Figure $1 B)\left(p=.005, R^{2}=0.47\right)$. Vasti, gastrocnemius and soleus contributions to AP GRFs did not predict braking asymmetry (Figure 1D-F), although the relationship between vasti contributions to braking and braking asymmetry did approach significance (Figure 1D) $\left(p=0.07, R^{2}=0.23\right)$.

Four out of fifteen stroke survivors $(27 \%)$ had greater plantarflexor contributions to propulsion on their paretic leg compared to their nonparetic leg (Table 3, see additional file). All four produced more braking with the paretic vasti than the nonparetic vasti. A total of seven out of fifteen stroke survivors $(47 \%)$ produced more braking with the paretic vasti than the nonparetic vasti. Two examples of muscles 
contributions to braking and propulsion are provided to illustrate propulsion asymmetries in opposite directions (Figure 2).

\section{Relationship between braking and propulsion and swing phase knee flexion}

There was moderate correlation between pre-swing AP GRF impulse and peak swing phase knee flexion (Figure 3A). Vasti contributions to braking in pre-swing relative to walking speed were correlated to knee flexion in swing, while soleus and gastrocnemius contributions to propulsion in pre-swing were not (Figure 3B-D).

\section{Primary contributors to knee flexion and extension acceleration in pre-swing}

The iliopsoas was the greatest contributor to pre-swing knee flexion acceleration (Figure 4). On average, individuals with low knee flexion had greater contributions to knee flexion acceleration in pre-swing from the iliopsoas. However, that result was primarily due to four individuals with very high iliopsoas contributions to knee flexion while the other five participants in the low knee flexion group had similar iliopsoas contributions as the typical knee flexion group (Figure 4). The rectus femoris was not a primary contributor to knee extension acceleration in pre-swing for any group. Rather, the vasti had a greater contribution to knee extension in the low knee flexion group compared to the typical knee flexion and control groups.

\section{Muscle contributions to leg swing initiation}

Knee flexion was not predicted by total muscle-tendon work performed on the paretic leg in pre-swing regardless of whether work was normalized by walking speed $\left(p=0.18, R^{2}=0.13\right.$ and $p=0.58, R^{2}=0.02$ for normalized and not normalized, respectively). The low knee flexion group did have lower total muscle contributions to paretic leg swing on average, but there was high variability between participants in how this was accomplished (Figure 5). On average, the low knee flexion group had lower leg swing contributions from the iliopsoas than the typical and control groups regardless of walking speed (Figure 5). Both the low and typical knee flexion groups had more power absorption from rectus femoris and vasti on average than the control group relative to their walking speeds (Figure 5A). However, there was significant variability in the low knee flexion group, with some participants having very low and some having very high power absorption from the rectus femoris.

\section{Discussion}

\section{Muscle contributions to braking and propulsion asymmetry}


The purpose of this study was to investigate impairments in early leg swing that may lead to stiff knee gait in individuals post-stroke. Specifically, we investigated pre-swing muscle contributions to braking, propulsion, knee flexion and leg swing initiation and the underlying relationships between pre-swing muscle function and swing phase knee kinematics in individuals post-stroke. We hypothesized that braking and propulsion asymmetry would correlate with both plantarflexor contributions to propulsion and excessive vasti contributions to braking. These hypotheses were partially supported. Gastrocnemius contributions to propulsion were correlated to propulsion symmetry. However, soleus and vasti contributions to AP GRF impulses were not correlated to braking or propulsion symmetry (Figure 1), although there was a strong trend towards an association between vasti and braking. These results are consistent with previous work showing that in individuals with moderate stroke, gastrocnemius and not soleus or vasti activity were correlated with AP GRF impulse [36]. Moreover, it is well established that the plantarflexor muscles are important rehabilitation targets for improving paretic propulsion and walking speed [9]. For example, Participant 15 produced more paretic propulsion than nonparetic propulsion ( $\mathrm{PP}=$ 0.71) but had lower net plantarflexor contributions to propulsion on the paretic side throughout stance, especially the soleus which did not contribute to propulsion, but instead produced braking in early and late stance (Figures 2C \& 2D), likely due to a lack of leg extension [37]. Participant 15 compensated for these low plantarflexor contributions to propulsion by relying on the hamstrings for propulsion (Figure 2CD). However, while paretic propulsion was high relative to the nonparetic leg, Participant 15 had low propulsion overall and thus walked slowly at $0.2 \mathrm{~m} / \mathrm{s}$ (Table 1), indicating that compensation from the hamstrings is not an effective way to produce propulsion. These results can be contrasted with a representative healthy participant (Figure 2E), who produced braking in early stance with the vasti and propulsion in late stance with the plantarflexors as expected. These results demonstrate that while plantarflexor function is an important predictor of propulsion, paretic propulsion can be attained through compensatory mechanisms, such as from the hamstrings, which cannot be identified through force plate measurements of braking and propulsion.

The lack of correlation between vasti braking and propulsion asymmetry does not mean that the vasti do not contribute to propulsion deficits in stroke survivors, as extended braking from the vasti was a factor in propulsion asymmetry for multiple participants. For example, Participant 2 produced less paretic propulsion than nonparetic propulsion ( $\mathrm{PP}=0.24$ ) due to prolonged braking from the vasti and rectus femoris despite producing more propulsion with the paretic plantarflexors (Figure 2A-B). Moreover, we previously showed that individuals with co-activation of the plantarflexors and other muscles such as the vasti had higher paretic braking but not lower propulsion [17], suggesting that propulsion asymmetries occurred not because of low plantarflexor contributions to propulsion but also because of excessive braking from other muscles. Thus, the knee extensor muscles can play a significant role in propulsion asymmetries, as they are primary contributors to braking [e.g. 38] and can become overactive post-stroke [39]. Vasti that are active late in stance should be an important target of rehabilitation, as they may affect both propulsion and knee flexion.

\section{Relationship between braking and propulsion and swing phase knee flexion}

Page 10/22 
We hypothesized that vasti and plantarflexor contributions to propulsion and braking in pre-swing would predict swing phase knee flexion. This hypothesis was partially supported. Overall, a lower pre-swing AP GRF impulse and greater vasti contributions to braking were correlated with less knee flexion in swing, but plantarflexor contributions were not (Figure 3). Similarly, [7] found that knee flexion in stroke survivors was predicted by late braking forces, but not net propulsive forces. These results can be explained by the fact that the vasti are primary contributors to both braking and knee extension and vasti activity is normally minimal in pre-swing for healthy individuals. However, in one sample of stroke survivors with stiff knee gait, $83 \%$ had inappropriately late vasti activity [40]. Other work has suggested that knee flexion deficits may be driven primarily by low ankle push off rather than knee extensors preventing knee flexion [2]. However, those conclusions were developed using a kinematic proxy of push off force (peak vertical acceleration of the malleolus marker), a methodological difference that may explain the differences in our studies. Moreover, it was concluded that individuals with lower knee flexion velocity than predicted by the malleolus acceleration model had stiff knee gait due to muscles preventing knee flexion rather than a low push-off force. However, it is likely that excess knee extensor activity could affect vertical malleolus acceleration and therefore these measures are not independent, leading some participants to be classified as having low knee flexion due to low push-off acceleration when knee extensor activity may have also contributed. In summary, our results suggest knee extensor activity in pre-swing predicts reduced knee flexion, while muscle contributions to propulsion in late stance do not.

\section{Primary contributors to knee flexion and extension in pre- swing}

We hypothesized that the rectus femoris would be a major contributor to knee extension in pre-swing in individuals with stiff knee gait. This hypothesis was not supported by our results. Rectus femoris contributions to knee extension were minimal in all groups (Figure 4), while the stiff knee group had greater knee extension contributions from the vasti. Previous work demonstrated that the while the gluteus maximus, vasti and rectus femoris had the greatest potential to accelerate the knee into extension during pre-swing, the gluteus maximus and rectus femoris produced significantly less force than the vasti in pre-swing, and produced less knee extension than the vasti and soleus, while the iliopsoas produced the most knee flexion [25]. Previous work has also proposed that low knee flexion could be due to weak hip flexor muscles, as hip flexors are key contributors to knee flexion in healthy gait $[22,41]$. Modeling studies have identified reduced iliopsoas function during pre-swing in individuals poststroke with poor walking function [5]. However, we observed that individuals in the low knee flexion group had greater iliopsoas contributions to knee flexion in pre-swing than healthy controls (Figure 4), although this average was dominated by four individuals, with others having lower than average iliopsoas contributions to knee flexion. This result may have occurred in part because the potential of the iliopsoas to flex the knee increases with reduced knee flexion. Interestingly, while [25], [41] and [15] found that the gastrocnemius contributed to knee flexion in double support, [11] found that the gastrocnemius contributed to knee extension in double support. The results of this study suggest that the gastrocnemius may perform both functions (Figure 4) depending on the individual's kinematic state. 
There is evidence that the rectus femoris does contribute to stiff knee gait, as rectus femoris hyperreflexia was found to be correlated to peak knee flexion angle [32]. However, Botox injections for stroke survivors with stiff knee gait have had mixed results [e.g. 42], with some individuals responding well and some not benefitting at all. Thus, there is a need to identify individuals who will benefit from treatments targeting the rectus femoris and others that may experience low knee flexion due to other problems such overactive vasti or weak hip flexors.

\section{Muscle contributions to leg swing initiation}

We hypothesized that greater knee flexion would be correlated with greater power delivered to the leg in pre-swing, but this hypothesis was not supported. While on average the low knee flexion group had less power delivered to the paretic leg in pre-swing compared to the typical knee flexion and control groups, there was significant variability between participants (Figure 5).

While the rectus femoris was not a primary contributor to knee extension in individuals with low knee flexion, it may still limit leg swing by absorbing power from the leg. Muscles act to either generate, absorb or transfer power between body segments $[34,43]$. In healthy individuals, the rectus femoris lengthens in late stance and absorbs power from the leg and redistributes it to the trunk [16]. Compared with healthy controls, a number of participants with low knee flexion had very high power absorption from the rectus femoris in pre-swing relative to their walking speed which would inhibit leg swing initiation (Figure 5), and thus limit the trajectory of knee flexion during swing.

The iliopsoas contributed less to leg swing initiation in both stroke groups on average compared healthy controls, but especially the low knee flexion group (Figure 5). These results are consistent with previous work showing lower power contributions from the iliopsoas in pre-swing for stroke survivors compared to healthy controls [14]. On average the gastrocnemius contributed less to leg swing initiation in the low knee flexion group compared to the typical knee flexion group (Figure 5), but contributed more to leg swing initiation in the typical knee flexion group when compared to healthy controls. These results of typical knee flexion group contrast previous work that found lower gastrocnemius contributions to leg swing initiation in stroke survivors [14]. However, that study only analyzed two representative stroke survivors, while ours shows that substantial variability exists between participants. The gastrocnemius may have contributed more to leg swing initiation in the stroke group with typical knee flexion due to the co-contraction seen in some participants (e.g. Participant 2), where greater plantarflexor output was required to overcome excessive braking from the vasti in late stance.

\section{Limitations}

A potential limitation of this study is that stroke survivors often have high levels of muscle co-contraction $[44,45]$, which may be difficult to replicate in simulations using algorithms such as computed muscle control which minimize the sum of muscle activations squared [30]. To address this concern, bilateral EMG was collected and muscle excitations were constrained to stay within bounds determined by experimentally collected EMG. Another limitation is that due to the high variability between individuals, it is difficult to generalize these results to the post-stroke population as a whole. Even with this limitation, 
few simulation studies of individuals post-stroke include a sample size as large as the present study, which demonstrates the variability between participants and highlights the many different causes of walking deficits. Future work will seek to develop clinical methods for identifying underlying causes of propulsion and leg swing deficits to inform individualized rehabilitation strategies. Another limitation is that this study focused only on muscle contributions to propulsion, while appropriate leg extension angle at push-off also affects propulsion [8, 9, 37]. It is likely that some of these participants experienced propulsion asymmetry in part due to leg positioning and future work should investigate causes of reduced leg extension. A final limitation is the interpretation of results could be affected by some control participants who had below average self-selected walking speeds and asymmetric walking. However, these controls were not used for statistical analyses and served to provide a reference for age-similar individuals without neurological injury rather than perfect walking.

\section{Conclusions}

We observed that some participants had paretic propulsion deficits due to low plantarflexor contributions to propulsion and/or excess vasti contributions to braking. Others appeared to produce sufficient or high paretic propulsion, but accomplished that propulsion via compensatory mechanisms such as a reliance on the hamstrings rather than appropriate plantarflexor activity. Greater vasti contributions to braking in pre-swing predicted lower knee flexion. The rectus femoris and iliopsoas did not directly contribute to lower flexion in pre-swing. However, in a number of cases the rectus femoris absorbed more power and the iliopsoas contributed less power from the paretic leg in pre-swing in individuals with low knee flexion. These results highlight the heterogeneity of the post-stroke population and the need to identify the underlying causes of propulsion and knee flexion deficits in each individual in order to improve rehabilitation outcomes.

\section{Abbreviations}

EMG

Electromyography

GRF

Ground reaction force

RRA

Residual reduction algorithm

COM

Center of mass

$\mathrm{CMC}$

Computed muscle control

PP

Percentage paretic propulsion

PB 
Percentage paretic braking

AP

Anteroposterior

\section{Declarations}

\section{Ethics approval and consent to participate}

The present study was approved by the Medical University of South Carolina Institutional Review Board. All participants provided written informed consent to participate.

\section{Consent for publication}

Not applicable.

\section{Availability of data and materials}

The datasets used in this study are available from the corresponding author on reasonable request.

\section{Competing interests}

The authors declare that they have no competing interests.

\section{Funding}

This work was supported in part by the National Science foundation Graduate Research Fellowship Program, NIH P20HD109040, VA RR\&D 1I01RX001935 and the Rehabilitation Research \& Development service of the VA.

\section{Author's contributions}

LB: Concept and design, analysis and interpretation of data, preparation of manuscript, SK: Concept and design, acquisition of data, editing of manuscript. RN: Concept and design, interpretation of data, editing of manuscript. All authors read and approved the final manuscript.

\section{Acknowledgements}


The authors would like to acknowledge Dr. Chris Gregory and Dr. Mark Bowden for their work collecting the data that was used in this study.

\section{References}

1. Virani SS, Alonso A, Benjamin EJ, Bittencourt MS, Callaway CW, Carson AP, et al. Heart Disease and Stroke Statistics-2020 Update: A Report From the American Heart Association. Circulation. 2020 Mar 3;141(9):e139-596.

2. Campanini I, Merlo A, Damiano B. A method to differentiate the causes of stiff-knee gait in stroke patients. Gait Posture. 2013 Jun;38(2):165-9.

3. Schmid A, Duncan PW, Studenski S, Lai SM, Richards L, Perera S, et al. Improvements in SpeedBased Gait Classifications Are Meaningful. Stroke. 2007 Jul 1;38(7):2096-100.

4. Bowden MG, Balasubramanian CK, Neptune RR, Kautz SA. Anterior-Posterior Ground Reaction Forces as a Measure of Paretic Leg Contribution in Hemiparetic Walking. Stroke. 2006 Mar 1;37(3):872-6.

5. Hall AL, Peterson CL, Kautz SA, Neptune RR. Relationships between muscle contributions to walking subtasks and functional walking status in persons with post-stroke hemiparesis. Clinical Biomechanics. 2011 Jun 1;26(5):509-15.

6. Kesar TM, Perumal R, Reisman DS, Jancosko A, Rudolph KS, Higginson JS, et al. Functional Electrical Stimulation of Ankle Plantarflexor and Dorsiflexor Muscles. Stroke. 2009 Dec 1;40(12):3821-7.

7. Dean JC, Bowden MG, Kelly AL, Kautz SA. Altered post-stroke propulsion is related to paretic swing phase kinematics. Clinical Biomechanics. 2020 Feb 1;72:24-30.

8. Awad LN, Lewek MD, Kesar TM, Franz JR, Bowden MG. These legs were made for propulsion: advancing the diagnosis and treatment of post-stroke propulsion deficits. Journal of NeuroEngineering and Rehabilitation. 2020 Oct 21;17(1):139.

9. Roelker SA, Bowden MG, Kautz SA, Neptune RR. Paretic propulsion as a measure of walking performance and functional motor recovery post-stroke: A review. Gait \& Posture. 2019 Feb 1;68:614.

10. McGowan CP, Kram R, Neptune RR. Modulation of leg muscle function in response to altered demand for body support and forward propulsion during walking. J Biomech. 2009 May 11;42(7):850-6.

11. Neptune RR, Kautz SA, Zajac FE. Contributions of the individual ankle plantar flexors to support, forward progression and swing initiation during walking. Journal of Biomechanics. 2001 Nov;34(11):1387-98.

12. Allen JL, Kautz SA, Neptune RR. Forward propulsion asymmetry is indicative of changes in plantarflexor coordination during walking in individuals with post-stroke hemiparesis. Clinical Biomechanics. 2014 Aug;29(7):780-6.

13. Nadeau S, Gravel D, Arsenault AB, Bourbonnais D. Plantarflexor weakness as a limiting factor of gait speed in stroke subjects and the compensating role of hip flexors. Clinical Biomechanics. 1999 Feb 
1;14(2):125-35.

14. Peterson CL, Hall AL, Kautz SA, Neptune RR. Pre-swing deficits in forward propulsion, swing initiation and power generation by individual muscles during hemiparetic walking. J Biomech. 2010 Aug 26;43(12):2348-55.

15. Knarr BA, Kesar TM, Reisman DS, Binder-Macleod SA, Higginson JS. Changes in the activation and function of the ankle plantar flexor muscles due to gait retraining in chronic stroke survivors. Journal of NeuroEngineering and Rehabilitation. 2013 Jan 31;10:12.

16. Neptune RR, Zajac FE, Kautz SA. Muscle force redistributes segmental power for body progression during walking. Gait \& Posture. 2004 Apr 1;19(2):194-205.

17. Brough LG, Kautz SA, Bowden MG, Gregory CM, Neptune RR. Merged plantarflexor muscle activity is predictive of poor walking performance in post-stroke hemiparetic subjects. Journal of Biomechanics. 2019 Jan;82:361-7.

18. Balaban B, Tok F. Gait Disturbances in Patients With Stroke. PM\&R. 2014 Jul 1;6(7):635-42.

19. Olney SJ, Richards C. Hemiparetic gait following stroke. Part I: Characteristics. Gait \& Posture. 1996 Apr 1;4(2):136-48.

20. Anderson FC, Goldberg SR, Pandy MG, Delp SL. Contributions of muscle forces and toe-off kinematics to peak knee flexion during the swing phase of normal gait: an induced position analysis. Journal of Biomechanics. 2004 May 1;37(5):731-7.

21. Goldberg SR, Õunpuu S, Delp SL. The importance of swing-phase initial conditions in stiff-knee gait. Journal of Biomechanics. 2003 Aug 1;36(8):1111-6.

22. Piazza SJ, Delp SL. The influence of muscles on knee flexion during the swing phase of gait. $J$ Biomech. 1996 Jun;29(6):723-33.

23. Tenniglo MJ, Nederhand MJ, Prinsen EC, Nene AV, Rietman JS, Buurke JH. Effect of Chemodenervation of the Rectus Femoris Muscle in Adults With a Stiff Knee Gait Due to Spastic Paresis: A Systematic Review With a Meta-Analysis in Patients With Stroke. Archives of Physical Medicine and Rehabilitation. 2014 Mar 1;95(3):576-87.

24. Reinbolt JA, Fox MD, Arnold AS, Ounpuu S, Delp SL. Importance of preswing rectus femoris activity in stiff-knee gait. J Biomech. 2008 Aug 7;41(11):2362-9.

25. Goldberg SR, Anderson FC, Pandy MG, Delp SL. Muscles that influence knee flexion velocity in double support: implications for stiff-knee gait. Journal of Biomechanics. 2004 Aug 1;37(8):1189-96.

26. Sangeux M, Polak J. A simple method to choose the most representative stride and detect outliers. Gait \& Posture. 2015 Feb 1;41(2):726-30.

27. Delp SL, Anderson FC, Arnold AS, Loan P, Habib A, John T, et al. OpenSim: Open-source software to create and analyze dynamic simulations of movement. IEEE Transactions on Biomedical Engineering. 2007;1940-1950.

28. Delp SL, Loan JP, Hoy MG, Zajac FE, Topp EL, Rosen JM. An interactive graphics-based model of the lower extremity to study orthopaedic surgical procedures. IEEE Transactions on Biomedical 
Engineering. 1990 Aug;37(8):757-67.

29. Hicks JL, Uchida TK, Seth A, Rajagopal A, Delp SL. Is my model good enough? Best practices for verification and validation of musculoskeletal models and simulations of movement. J Biomech Eng. 2015 Feb 1;137(2):020905.

30. Thelen DG, Anderson FC. Using computed muscle control to generate forward dynamic simulations of human walking from experimental data. J Biomech. 2006;39(6):1107-15.

31. Roelker SA, Kautz SA, Neptune RR. Muscle contributions to mediolateral and anteroposterior foot placement during walking. Journal of Biomechanics. 2019 Oct;95:109310.

32. Akbas T, Kim K, Doyle K, Manella K, Lee R, Spicer P, et al. Rectus femoris hyperreflexia contributes to Stiff-Knee gait after stroke. Journal of NeuroEngineering and Rehabilitation. 2020 Aug 26;17(1):117.

33. Sulzer JS, Gordon KE, Dhaher YY, Peshkin MA, Patton JL. Preswing Knee Flexion Assistance Is Coupled With Hip Abduction in People With Stiff-Knee Gait After Stroke. Stroke. 2010 Aug 1;41(8):1709-14.

34. Fregly BJ, Zajac FE. A state-space analysis of mechanical energy generation, absorption, and transfer during pedaling. Journal of Biomechanics. 1996 Jan 1;29(1):81-90.

35. Hamner SR, Seth A, Delp SL. Muscle contributions to propulsion and support during running. J Biomech. 2010 Oct 19;43(14):2709-16.

36. Turns LJ, Neptune RR, Kautz SA. Relationships between muscle activity and anteroposterior ground reaction forces in hemiparetic walking. Arch Phys Med Rehabil. 2007 Sep;88(9):1127-35.

37. Peterson CL, Cheng J, Kautz SA, Neptune RR. Leg extension is an important predictor of paretic leg propulsion in hemiparetic walking. Gait \& Posture. 2010 Oct;32(4):451-6.

38. Liu MQ, Anderson FC, Pandy MG, Delp SL. Muscles that support the body also modulate forward progression during walking. Journal of Biomechanics. 2006 Jan 1;39(14):2623-30.

39. Yelnik A, Albert T, Bonan I, Laffont I. A Clinical Guide to Assess the Role of Lower Limb Extensor Overactivity in Hemiplegic Gait Disorders. Stroke. 1999 Mar 1;30(3):580-5.

40. Kerrigan DC, Gronley J, Perry J. STIFF-LEGGED GAIT IN SPASTIC PARESIS A Study of Quadriceps and Hamstrings Muscle Activity. American Journal of Physical Medicine \& Rehabilitation. 1991 Dec;70(6):294-305.

41. Yamaguchi GT, Zajac FE. Restoring unassisted natural gait to paraplegics via functional neuromuscular stimulation: a computer simulation study. IEEE Transactions on Biomedical Engineering. 1990 Sep;37(9):886-902.

42. Caty GD, Detrembleur C, Bleyenheuft C, Deltombe T, Lejeune TM. Effect of Simultaneous Botulinum Toxin Injections Into Several Muscles on Impairment, Activity, Participation, and Quality of Life Among Stroke Patients Presenting With a Stiff Knee Gait. Stroke. 2008 Oct 1;39(10):2803-8.

43. Zajac FE, Neptune RR, Kautz SA. Biomechanics and muscle coordination of human walking: Part I: Introduction to concepts, power transfer, dynamics and simulations. Gait \& Posture. 2002;16(3):21532. 
44. Clark DJ, Ting LH, Zajac FE, Neptune RR, Kautz SA. Merging of Healthy Motor Modules Predicts Reduced Locomotor Performance and Muscle Coordination Complexity Post-Stroke. J Neurophysiol. 2010 Feb;103(2):844-57.

45. Lamontagne A, Richards CL, Malouin F. Coactivation during gait as an adaptive behavior after stroke. J Electromyogr Kinesiol. 2000 Dec;10(6):407-15.

Figures

(A)

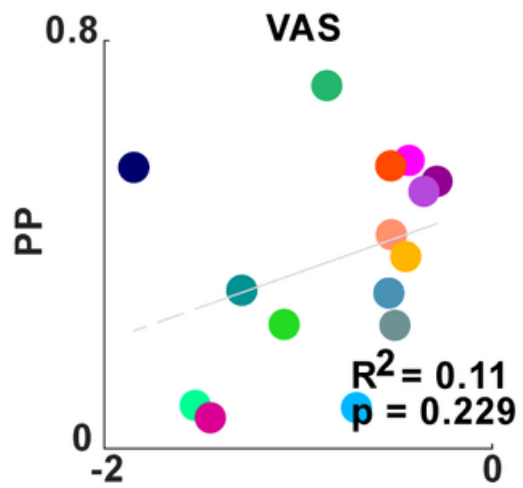

(B)

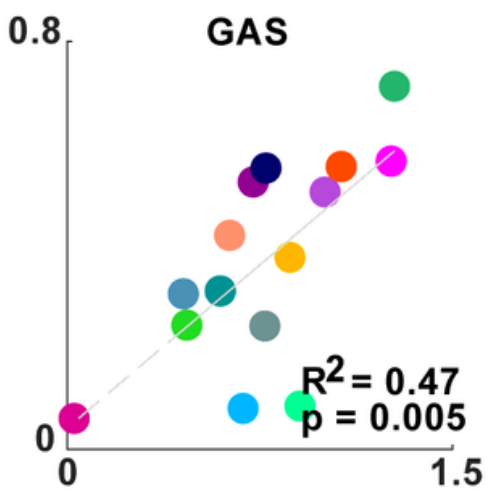

Dimensionless AP GRF Impulse

(C)

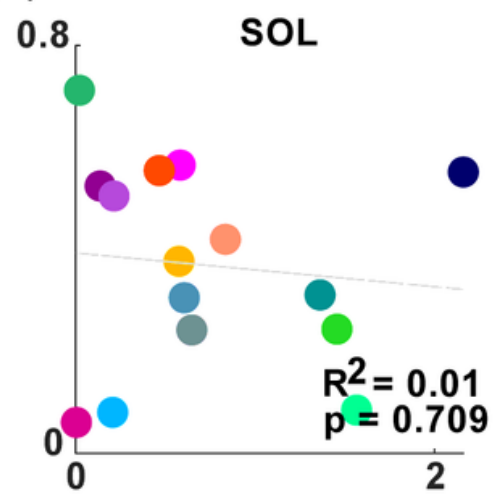

Braking Asymmetry

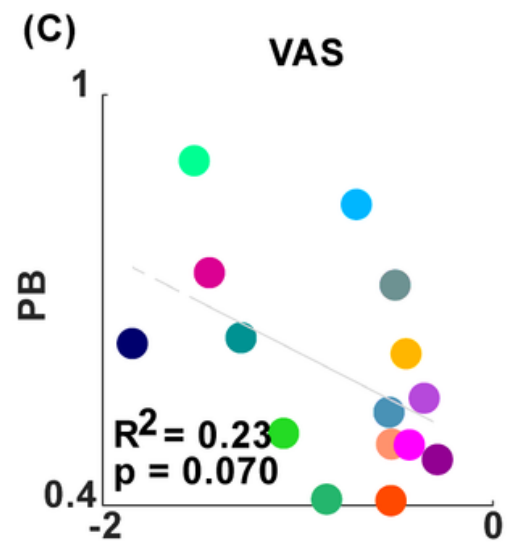

(D)

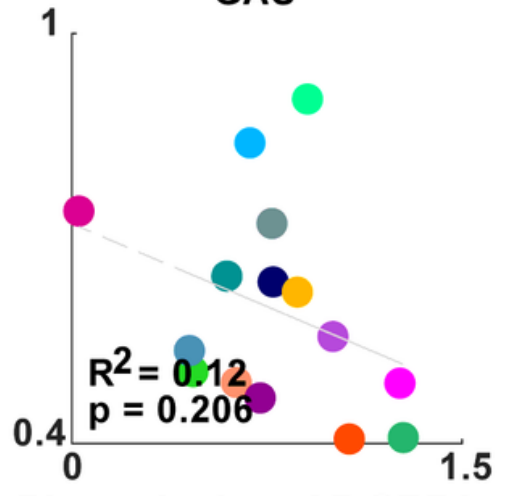

Dimensionless AP GRF Impulse

(E)
Subject 1

Subject 2

Subject 3

Subject 4

Subject 5

Subject 6

Subject 7

Subject 8

Subject 9

SOL

Subject 10

Subject 11

Subject 12

Subject 13

Subject 14

Subject 15

\section{Figure 1}

Predictors of Propulsion and Braking Asymmetry. Correlation between percentage paretic propulsion (PB) and percentage paretic braking $(\mathrm{PB})$ and muscle contributions to propulsion and braking relative to walking speed in stroke survivors. 
Subject 2

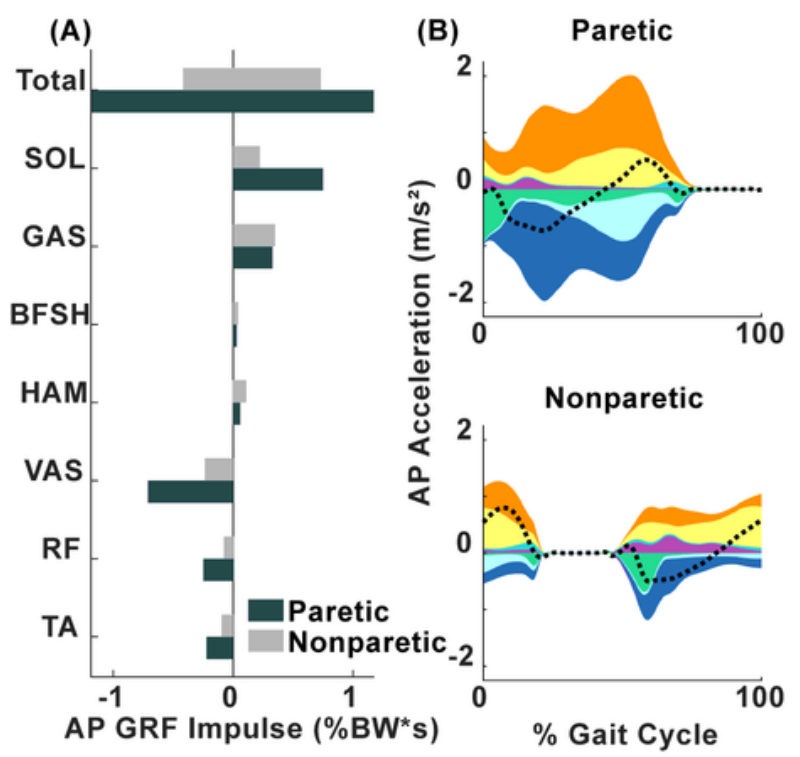

\section{Subject 15}
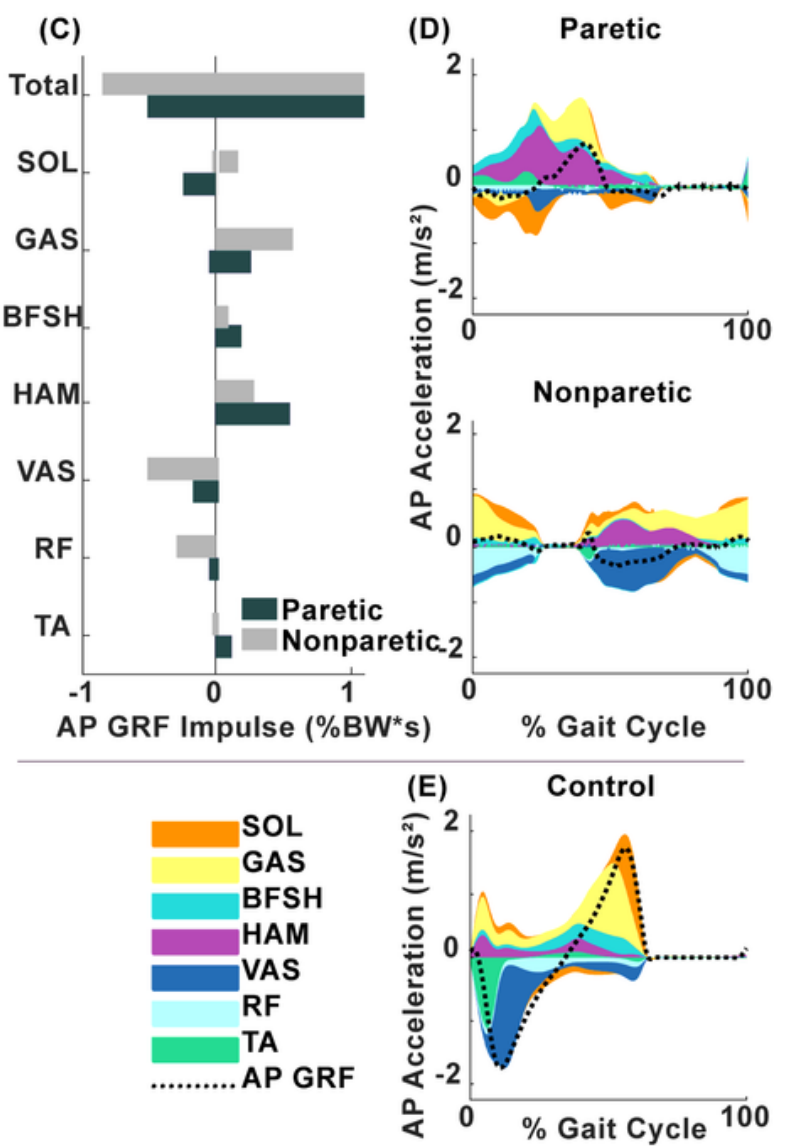

Figure 2

Muscle Contributions to Braking and Propulsion. A) Paretic and nonparetic muscle contributions to AP COM acceleration integrated over stance for Subject 2. B) Muscle contributions to AP COM acceleration over the paretic gait cycle, with contributions stacked on top of one another and shown relative to the normalized AP GRF (dotted line) for Subject 2. C) Paretic and nonparetic muscle contributions to AP COM acceleration integrated over stance for Subject 15. D) Muscle contributions to AP COM acceleration over 
the paretic gait cycle, with contributions stacked on top of one another and shown relative to the normalized AP GRF (dotted line) for Subject 15. E) Muscle contributions to AP COM acceleration over the left gait cycle, with contributions stacked on top of one another and shown relative to the normalized AP GRF (dotted line) for a representative control subject.

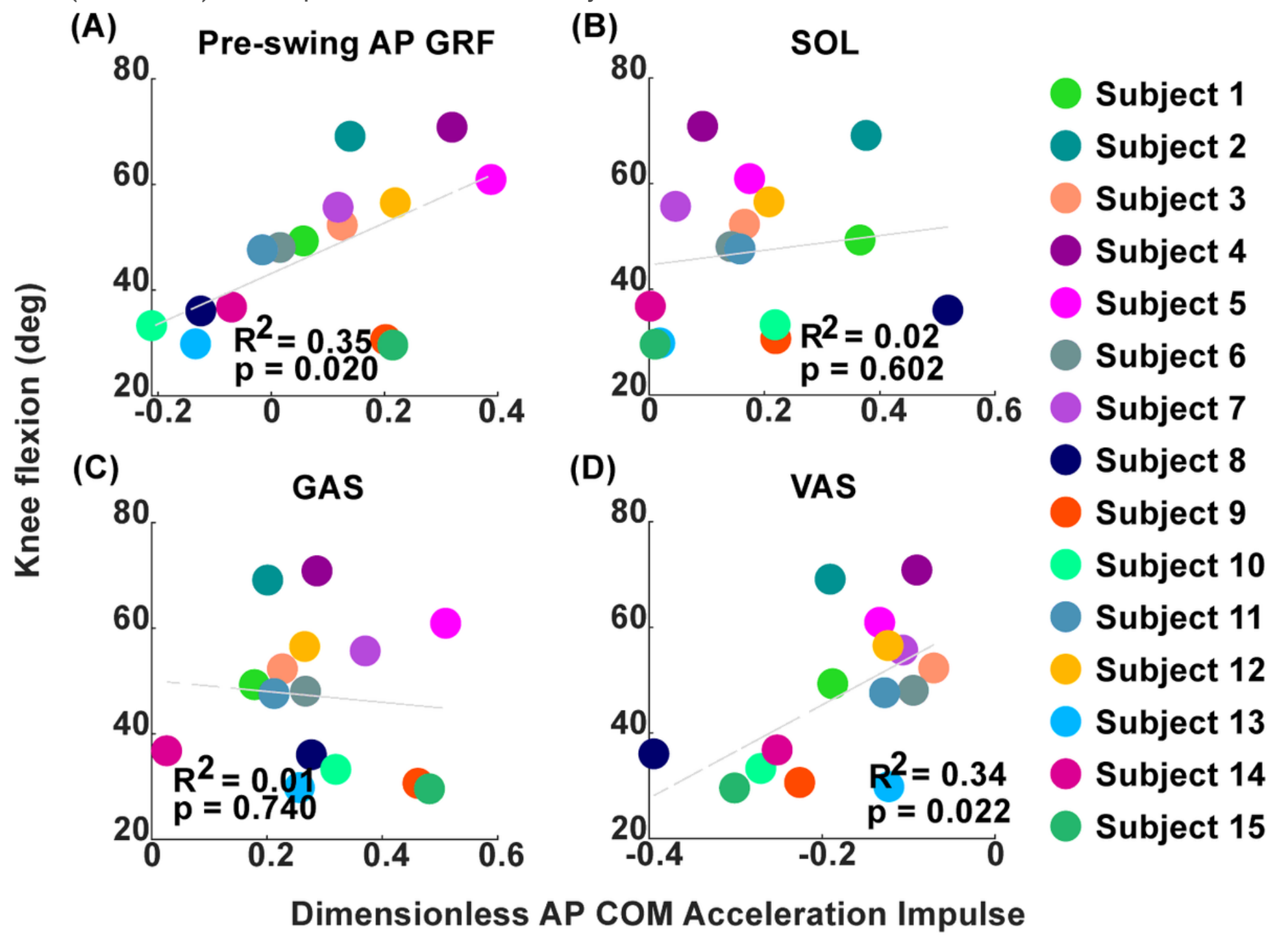

Figure 3

Predictors of Peak Knee Flexion. Correlation between peak knee flexion during swing and potential predictors of knee flexion, including $A$ ) the impulse of paretic AP GRFs in pre-swing normalized by subject mass, B-D) The AP COM acceleration impulse in pre-swing contributed by the SOL, GAS and VAS groups. 


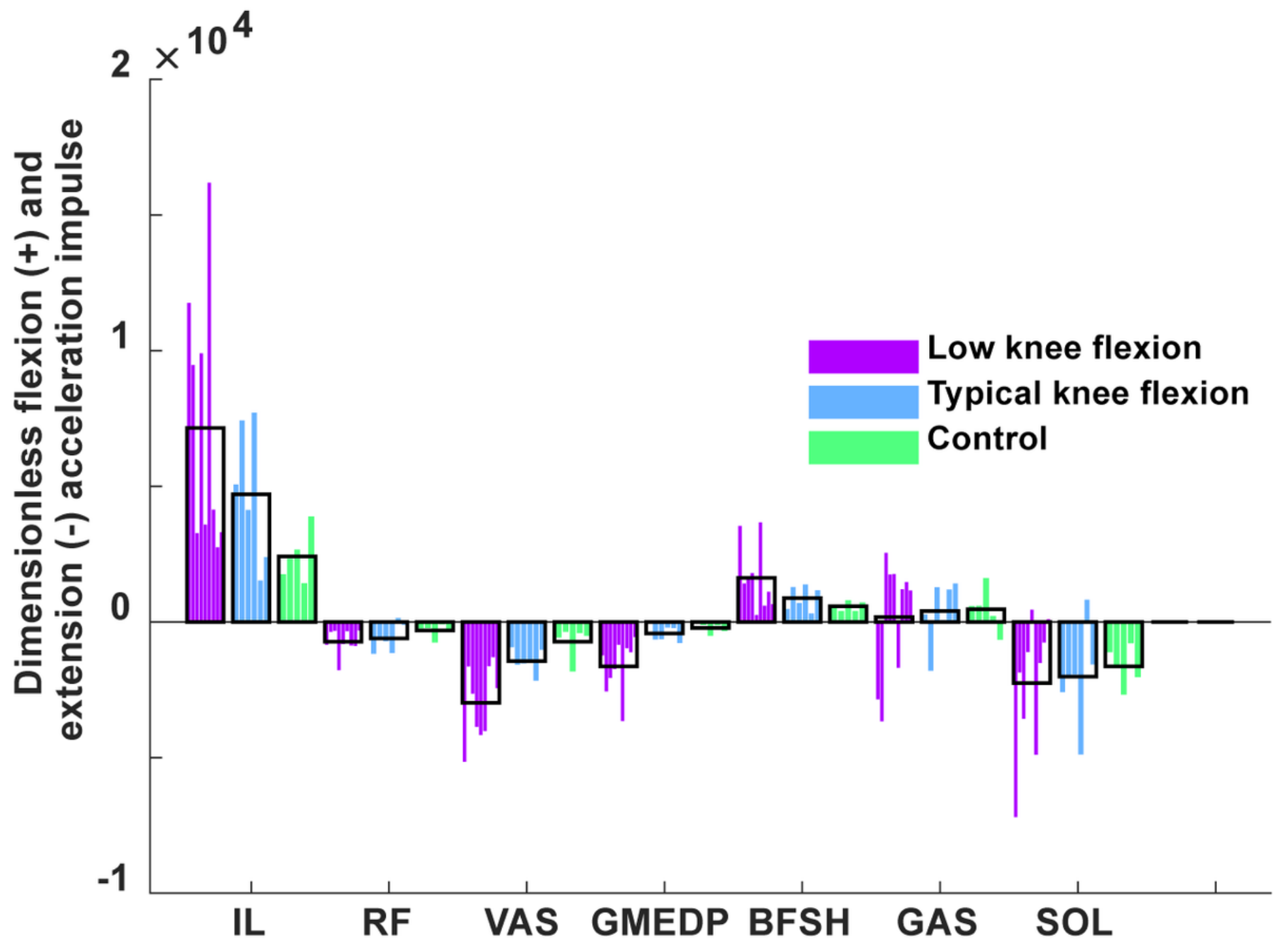

Figure 4

Muscle Contributions to Knee Flexion in Pre-Swing. Muscle contributions to knee flexion and extension acceleration integrated over pre-swing and normalized by walking speed. Participants are ordered from least to greatest knee flexion during swing. 
(A)

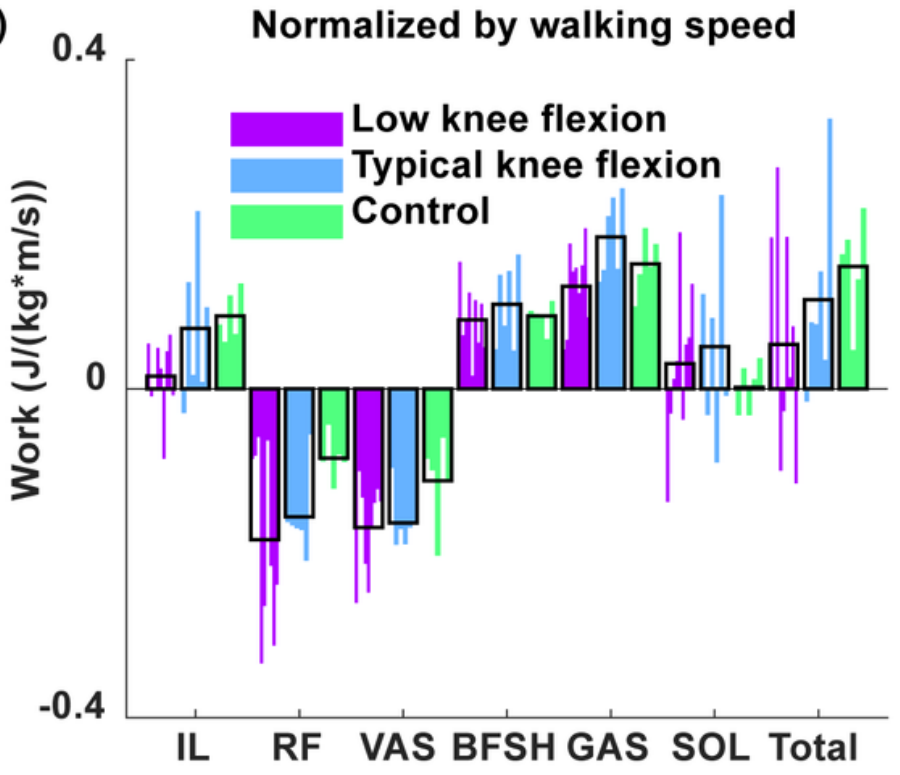

(B)

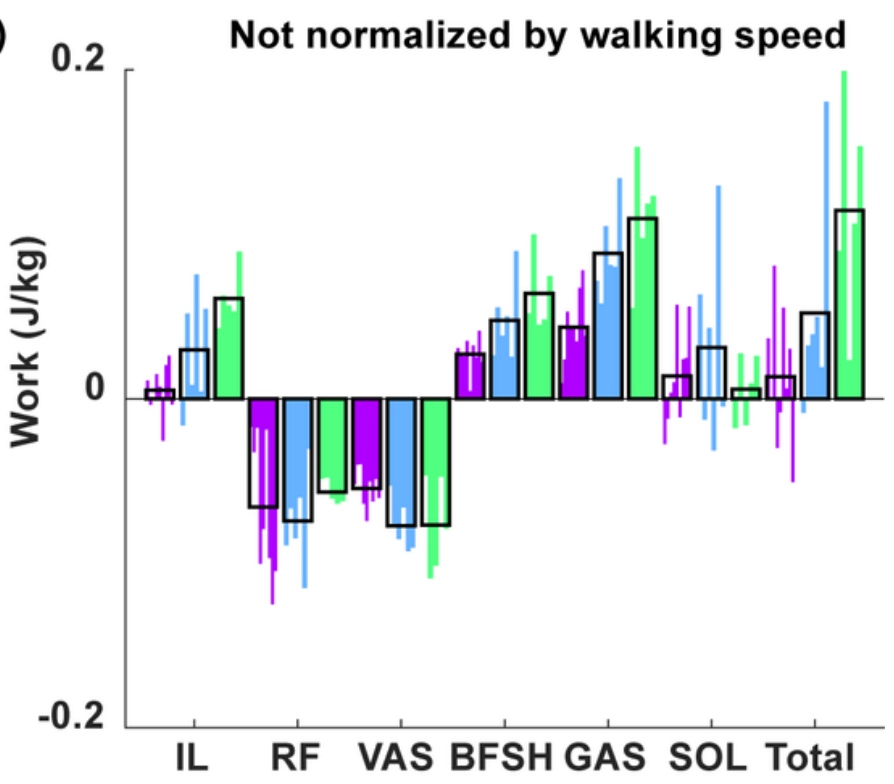

Figure 5

Muscle Contributions to Leg Swing Initiation. Work performed on the paretic leg in pre-swing A) normalized by walking speed, and B) not normalized by walking speed. Participants are ordered from least to most knee flexion during swing.

\section{Supplementary Files}

This is a list of supplementary files associated with this preprint. Click to download.

- AdditionalFile1.pdf 